\title{
Longterm Outcomes of Temporomandibular Joints in Juvenile Idiopathic Arthritis: 17 Years of Followup of a Nordic Juvenile Idiopathic Arthritis Cohort
}

\author{
Mia Glerup (iD), Peter Stoustrup, Louise H. Matzen, Veronika Rypdal, Ellen Nordal, Paula Frid, Ellen Dalen Arnstad, \\ Marite Rygg, Olafur Thorarensen, Maria Ekelund, Lillemor Berntson, Anders Fasth, Håkan Nilsson, Suvi Peltoniemi, \\ Kristiina Aalto, Sirpa Arte, Peter Toftedal, Susan Nielsen, Sven Kreiborg (iD), Troels Herlin, and Thomas Klit Pedersen
}

\begin{abstract}
Objective. To determine the prevalence of orofacial symptoms, dysfunctions, and deformities of the temporomandibular joint (TMJ) in juvenile idiopathic arthritis (JIA) 17 years after disease onset.

Methods. Drawn from a prospective, population-based Nordic JIA cohort with disease onset from 1997 to 2000, 420 consecutive cases were eligible for orofacial evaluation of TMJ involvement. The followup visit included demographic data, a standardized clinical orofacial examination, and full-face cone-beam computed tomography (CBCT). For comparison, 200 age-matched healthy controls were used.

Results. Of 420 eligible participants with JIA, 265 (63\%) were included (mean age $23.5 \pm 4.2$ yrs) and completed a standardized clinical orofacial examination. Of these, 245 had a full-face CBCT performed. At least 1 orofacial symptom was reported by 33\%. Compared to controls, the JIA group significantly more often reported TMJ pain, TMJ morning stiffness, and limitation on chewing. Further, among participants reporting complaints, the number of symptoms was also higher in JIA. The mean maximal incisal opening was lower in the JIA group $(\mathrm{p}<0.001)$, and TMJ pain on palpation was more frequent. Condylar deformities and/or erosions were observed in $61 \%$ as assessed by CBCT, showing bilateral changes in about $70 \%$. Risk factors of condylar deformities were orofacial dysfunction or biologic treatment; enthesitis-related arthritis was protective.

Conclusion. This study of the longterm consequences of TMJ involvement in a population-based JIA cohort reports persistence of comprehensive symptoms, dysfunctions, and damage of the TMJ into adulthood. We suggest interdisciplinary followup of JIA patients also in adulthood. (First Release February 15 2020; J Rheumatol 2020;47:730-8; doi:10.3899/jrheum.190231)
\end{abstract}

Key Indexing Terms:

TEMPOROMANDIBULAR JOINT JUVENILE IDIOPATHIC ARTHRITIS OUTCOMES

\begin{abstract}
From the Department of Pediatrics, Aarhus University Hospital; Section of Orthodontics, Aarhus University; Section of Oral Radiology, Department of Dentistry and Oral Health, Aarhus University, Aarhus, Denmark; Department of Pediatrics, University Hospital of North Norway, Troms $\phi$; Department of Clinical Medicine, Faculty of Health Sciences, UiT The Arctic University of Norway, Troms $\phi$; Department of Otorhinolaryngology and Department and Division of Oral and Maxillofacial Surgery, University Hospital North Norway and Public Dental Service Competence Center of North Norway, Troms $\phi$; Department of Clinical and Molecular Medicine, Faculty of Medicine and Health Sciences, Norwegian University of Science and Technology (NTNU), Trondheim; Department of Pediatrics, Levanger Hospital, Nord-Trøndelag Hospital Trust, Levanger; Department of Pediatrics, St. Olavs Hospital, Trondheim; Department of Oral and Craniomaxillofacial Surgery, St. Olavs Hospital, Trondheim, Norway; Department of Pediatrics, Ryhov County Hospital, Jönköping; Department of Women's and Children's Health, Uppsala University, Uppsala; Department of Pediatrics, Sahlgrenska Academy, University of Gothenburg, Gothenburg; Department of Oral and Maxillofacial Surgery/Stomatognathic Physiology, The Institute for Postgraduate Dental Education, Jönköping, Sweden; Hospital for Children and Adolescents, University of Helsinki, Helsinki; Orthodontics, Oral and Maxillofacial Diseases, University of Helsinki and Helsinki University Hospital, Helsinki, Finland; Department of Pediatrics, Copenhagen University Hospital, Copenhagen; Department of Pediatric Dentistry and Clinical Genetics, University of Copenhagen, Copenhagen; Department of Oral and Maxillofacial Surgery, Aarhus University Hospital, Aarhus, Denmark. M. Glerup, MD, Department of Pediatrics, Aarhus University Hospital;
\end{abstract}

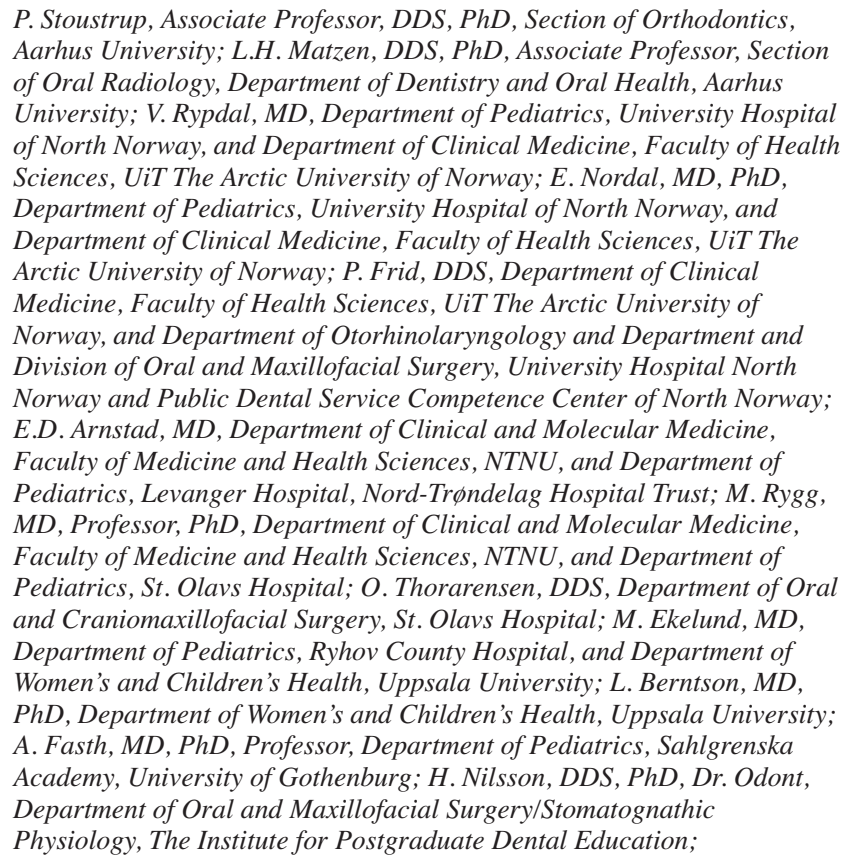

P. Stoustrup, Associate Professor, DDS, PhD, Section of Orthodontics, Aarhus University; L.H. Matzen, DDS, PhD, Associate Professor, Section of Oral Radiology, Department of Dentistry and Oral Health, Aarhus University; V. Rypdal, MD, Department of Pediatrics, University Hospital of North Norway, and Department of Clinical Medicine, Faculty of Health Sciences, UiT The Arctic University of Norway; E. Nordal, MD, PhD, Department of Pediatrics, University Hospital of North Norway, and Department of Clinical Medicine, Faculty of Health Sciences, UiT The Arctic University of Norway; P. Frid, DDS, Department of Clinical Medicine, Faculty of Health Sciences, UiT The Arctic University of Norway, and Department of Otorhinolaryngology and Department and Division of Oral and Maxillofacial Surgery, University Hospital North Norway and Public Dental Service Competence Center of North Norway; E.D. Arnstad, MD, Department of Clinical and Molecular Medicine, Faculty of Medicine and Health Sciences, NTNU, and Department of Pediatrics, Levanger Hospital, Nord-Trøndelag Hospital Trust; M. Rygg, MD, Professor, PhD, Department of Clinical and Molecular Medicine, Faculty of Medicine and Health Sciences, NTNU, and Department of Pediatrics, St. Olavs Hospital; O. Thorarensen, DDS, Department of Oral and Craniomaxillofacial Surgery, St. Olavs Hospital; M. Ekelund, MD, Department of Pediatrics, Ryhov County Hospital, and Department of Women's and Children's Health, Uppsala University; L. Berntson, MD, PhD, Department of Women's and Children's Health, Uppsala University; A. Fasth, MD, PhD, Professor, Department of Pediatrics, Sahlgrenska Academy, University of Gothenburg; H. Nilsson, DDS, PhD, Dr. Odont, Department of Oral and Maxillofacial Surgery/Stomatognathic Physiology, The Institute for Postgraduate Dental Education; 
Arthritis of the temporomandibular joint (TMJ) is a well-recognized entity in patients with juvenile idiopathic arthritis (JIA), with a prevalence of 40-86\% 1,2,3,4,5 depending on the cohort and imaging used ${ }^{6}$. A prime concern in relation to childhood TMJ arthritis is the development of temporomandibular disorders (TMD) and dentofacial deformities. These deformities develop as TMJ inflammation affects the condylar growth cartilage uniquely positioned intraarticularly ${ }^{7}$. Retrognathia developing into micrognathia is a common finding. Unilateral TMJ involvement may cause facial asymmetry ${ }^{8,9,10}$, and arthritis-induced orofacial symptoms or dysfunctions may impair patients' quality of life $2,3,11$.

Longterm ( $>10 \mathrm{yrs}$ ) outcomes of TMJ arthritis in JIA have been investigated in 4 cohorts $^{4,12,13,14}$, all from the prebiologic era. However, these cohorts have been small, selection-biased, and difficult to compare because of different treatment approaches. Hence, longterm orofacial manifestations of JIA in a nonselected JIA cohort in the biologic era remain unknown.

Using a population-based cohort with a 17-year followup, our aims were to (1) estimate the prevalence of orofacial symptoms and dysfunction in JIA compared to age-matched healthy controls; (2) estimate the prevalence of TMJ deformities assessed by cone-beam computed tomography (CBCT); and (3) suggest predictors for developing radiological TMJ deformities.

\section{MATERIALS AND METHODS}

Study design. We enrolled eligible JIA subjects from the Nordic JIA cohort 17 years after JIA onset. The cohort consists of consecutive cases with JIA diagnosed by experienced pediatric rheumatologists from particular geographical areas of Denmark, Finland, Norway, and Sweden. Characteristics of the cohort have been described ${ }^{15,16}$. The inclusion period was defined as disease onset from January 1, 1997, to June 30, 2000. Initially and during the inclusion period, letters were distributed to all referral institutions (primary care physicians, orthopedic surgeons, and pediatricians) in the catchment areas to ensure inclusion of all potential JIA candidates. The baseline visit took place 6 months $(-1 /+2$ months $)$ after disease onset, which was defined as the date of the first symptoms of arthritis described by the patient/parents/doctor. The date of diagnosis was the date the pediatric rheumatologist diagnosed JIA.

Registration of the active joints including the TMJ was done at baseline

S. Peltoniemi, MD, Hospital for Children and Adolescents, University of Helsinki; K. Aalto, MD, PhD, Hospital for Children and Adolescents, University of Helsinki; S. Arte, DDS, PhD, Orthodontics, Oral and Maxillofacial Diseases, University of Helsinki and Helsinki University Hospital; P. Toftedal, MD, PhD, Department of Pediatrics, Copenhagen University Hospital; S. Nielsen, MD, Department of Pediatrics, Copenhagen University Hospital; S. Kreiborg, Professor, DDS, PhD, Dr. Odont, Department of Pediatric Dentistry and Clinical Genetics, University of Copenhagen; T. Herlin, Professor, MD, DMSc, Department of Pediatrics, Aarhus University Hospital; T.K. Pedersen, Professor, DDS, $P h D$, Section of Orthodontics, Aarhus University, and Department of Oral and Maxillofacial Surgery, Aarhus University Hospital.

Address correspondence to Dr. M. Glerup, Department of Pediatrics, Aarhus University Hospital, Skejby, Palle Juul-Jensens Blvd. 99, 8200 Aarhus N, Denmark.E-mail: miagleru@rm.dk

Accepted for publication September 3, 2019. and prospectively ${ }^{15,16}$. To ensure a nonselected setting, all eligible patients were invited to participate regardless of their disease course. Last followup visit included an update on demographic data, standardized clinical orofacial examination according to consensus-based international recommendations ${ }^{17}$, and a CBCT. Originally, 510 patients with JIA were included, but some centers had no access to CBCT. They were therefore excluded from our present study (Figure 1). Their exclusion did not hamper the population-based approach of the study.

Inclusion and exclusion criteria. Participants fulfilling the International League of Associations for Rheumatology criteria of JIA ${ }^{18}$ and having had at least 2 followup visits were included. Among the exclusion criteria were having a cleft lip/palate or other dentofacial and craniofacial anomalies unrelated to arthritis.

Disease activity. The validated Juvenile Arthritis Disease Activity Score (JADAS) was used to assess disease activity. JADAS71 includes a physician's global assessment of disease activity (PGA; 0-10), patient's global assessment (PtGA) of general well-being (0-10), an active joint count assessed in 71 joints, and erythrocyte sedimentation rate (ESR) ${ }^{19}$ with a range of $0-101$ where $\leq 1$ is equivalent to inactive disease ${ }^{20}$. We applied the preliminary remission criteria of Wallace, et $\mathrm{al}^{21}$.

Definitions of TMJ involvement. We used the terminology of the standardized, consensus-based recommendations by the Temporomandibular Joint Juvenile Arthritis Work (TMJaw) Group 22 .

Assessment of orofacial symptoms. According to the international recommendations on orofacial examination in JIA ${ }^{17}$, the participants completed a Web-based questionnaire on orofacial pain frequency, intensity, location, and jaw function within the past 2 weeks. TMJ stiffness referred to morning stiffness in the jaw muscles and joints. Orofacial muscle pain was pain in the masseter and temporalis muscles, and chewing limitations when the participants avoided hard or chewy foods because of pain in the face or jaw. Pain frequency was defined as pain from the jaw area within the past 2 weeks categorized as (1) never (0 points); (2) not every week (1 point); (3) several times a week (2 points); (4) several times a day (3 points); and (5) all the time (4 points).

World Health Organization guidelines on cross-cultural translation and questionnaire adaptation were used ${ }^{23}$ for translation of the questionnaire to the 4 Scandinavian languages.

Assessment of dentofacial dysfunction. Standardized clinical examinations ${ }^{17}$ were performed by 8 specially trained investigators ( 5 orthodontists, 1 orofacial pain specialist, 1 pediatric dentistry specialist, and 1 oral maxillofacial surgeon).

The maximal incisal opening (MIO) was assessed as the distance between the lower and upper right incisal edge accounting for the vertical incisal overlap. Reduced mouth opening was defined as MIO $\leq-2$ SD below the mean of age-related normative values of 17 -year-olds ${ }^{24}$. The same type of ruler was used in all centers. TMJ pain on palpation was documented on a closed and open mouth position with index finger placed on the TMJ. Asymmetric mouth opening was defined as a mandibular deviation $\geq 3 \mathrm{~mm}$ to either side in relation to the vertical midface reference line at maximum mouth opening.

Radiographic assessment. CBCT examinations were performed using the manufacturer's recommendation on details regarding doses, radiation time, and voxel size. Standardization was optimized using the following criteria: (1) teeth in habitual occlusion with no protrusion of the mandible; and (2) a large field of view, at least 12 inches.

The radiological appearance of the condylar head was scored as normal (condylar shape with smooth, intact outline) or abnormal, assessing CBCT volumes in the lateral, coronal, and axial planes. Abnormal condyles were subcategorized based on the predominant radiologic feature: (1) deformity of the condyle with marked flattening or changes in shape with smooth and intact outline; (2) erosion of the condyle with disruption of the outline due to cysts or erosions; or (3) deformity plus erosive changes. Importantly, this scoring system of the osseous deformities does not refer to a scale with

Personal non-commercial use only. The Journal of Rheumatology Copyright @ 2020 . All rights reserved. 


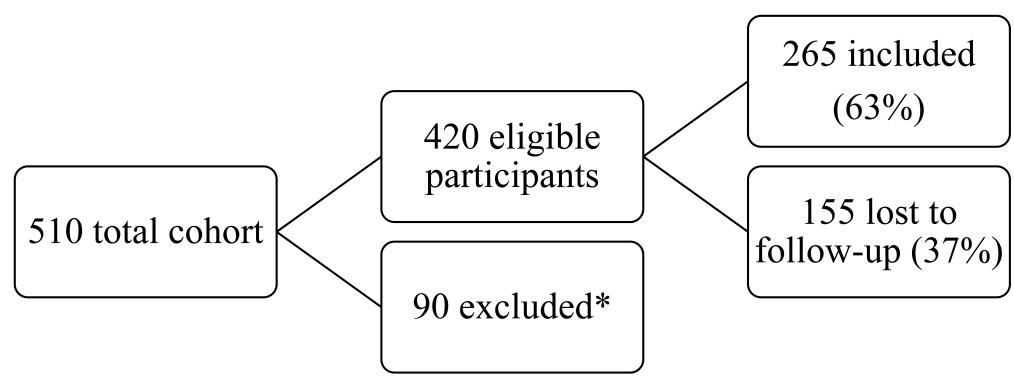

Figure 1. Flow chart of the study population. * Excluded were 6 of the 7 Swedish centers because they had no access to cone-beam computed tomography.

gradual progression. The same observer (LHM), who is highly experienced in assessing CBCT, performed the evaluations of the condyles in the CBCT volumes. LHM was blinded to all clinical and demographic data.

Control group. Nonselected Danish age-matched individuals $(\mathrm{n}=200)$ between 18 and 30 years were used for comparison. The control group was composed of young healthy adults (dental students, members of a sports club, and trainees within the retail industry) who voluntarily participated. Inclusion criteria for the controls were healthy individuals with no history of arthritis, osteoarthritis, cleft lip/palate or other craniofacial anomalies, or ongoing orthodontic treatment. Orofacial symptoms were reported in the same Web-based questionnaire as the JIA group, and the same standardized orofacial examination was used ${ }^{17}$. For ethical reasons, no CBCT examination was performed on the healthy controls.

Intraobserver variation in the assessment of the CBCT scans. Thirty CBCT (60 TMJ) examinations were assessed twice, 6 months apart, by the same observer (LHM) to test the intraobserver reliability.

Ethics. Approval was granted by national research committees in all the countries (1-10-72-280-13, 2012/2051, Dnr 2014/413-31, 174/13/03/03/2014), and the participants gave their written consent. Institutional Review Board approval was granted.

Statistics. Descriptive statistics of normally distributed data (mean \pm SD) and nonparametric data (median/interquartile range) were applied to assess the clinical characteristics of the cohort and disease activity. Chi-square test was conducted to compare dichotomous data. Mann-Whitney test and standardized test statistics reported as a $\mathrm{Z}$ score were used to compare medians on ordinal data. A logistic regression model was used to assess baseline predictors and predictors for the development of condylar changes. All candidate variables were dichotomized and OR calculated using a multivariate logistic regression model. Age at onset and sex were included in the model, and the level of significance was $\mathrm{p}<0.05$. Further, to identify high-risk participants at the 17 -year followup, we performed a logistic regression analysis of treatment, symptoms, dysfunctions, and JIA category.

Cohen's $\kappa$ coefficient was analyzed to test the interrater agreement for categorical measurements.

\section{RESULTS}

Study population. Of 420 eligible participants from the JIA cohort, $265(63.1 \%)$ were included in the present study (mean age $23.5 \pm$ SD $4.2 \mathrm{yrs}$; Figure 1). One patient was excluded because of fracture of a condyle. The mean followup time from JIA onset to orofacial examination was 17.3 years (SD $\pm 1.3 \mathrm{yrs}) ; 186 / 265(70.2 \%)$ were girls. The distribution of JIA categories and other clinical data were as described in Table 1. We found no difference in sex, JIA category, number of active joints, or baseline JADAS values between included participants and those lost to followup. However, age at onset was lower in the included group (mean $6.0 \pm 3.9$ vs $6.2 \pm 4.0$ yrs in those lost to followup; $\mathrm{p}=0.003)$. Of the 265 participants completing the clinical orofacial examination, 245 had a full-face CBCT performed with 490 approved high-quality TMJ images.

The control group had a mean age of $23.6 \pm 2.9$ years; $52.5 \%$ were girls.

Orofacial symptoms. In total, 87 of the 265 JIA participants $(32.8 \%)$ reported at least $1 \mathrm{TMJ}$-related symptom at the followup visit, which was similar to the control group $(p=0.11$; Table 2). Orofacial pain frequency and number of symptoms, TMJ pain, morning stiffness, and limitation of chewing were observed significantly more frequently in the JIA group and in all categories (Table 2). We found no intergroup difference in pain in the temporal $(\mathrm{p}=0.51)$ or masseter area $(\mathrm{p}=0.17$; data not shown). Overall, $89(33.6 \%)$ participants with JIA reported jaw and/or facial pain within the past 2 weeks. Of the controls, $61(30.5 \%)$ reported jaw and/or facial pain within the past 2 weeks, which was similar to the reports in the JIA group ( $\mathrm{p}=0.48)$, but more controls reported pain less than once a week ( $71 \%$ vs $53 \%)$ and fewer reported pain several times per day or all the time (7\% vs $27 \%$ ) compared to the JIA group.

Dysfunction. In total, 136 (51.3\%) participants with JIA had at least 1 clinical sign of orofacial dysfunction, not different from controls $(\mathrm{p}=0.12$; Table 2). The most frequent clinical findings were TMJ and orofacial pain on palpation in both groups. Mean MIO was significantly lower in the JIA group $(47.2 \pm 7.7$ vs $56.5 \pm 6.8 \mathrm{~mm} ; \mathrm{p}<0.001)$. The prevalence of participants with an $\mathrm{MIO}<-2 \mathrm{SD}$ was significantly higher among JIA participants than controls $(\mathrm{p}<0.001$; Table 2$)$. TMJ pain on palpation was more frequent in the JIA group than in controls $(p=0.03)$. However, no intergroup differences were observed for orofacial pain on palpation or asymmetric mouth opening.

Disease status and clinical findings. Inactive disease was found in $162(61.1 \%)$ of the 265 participants with JIA, of whom $34(12.8 \%)$ were in remission with medication, and 99 $(37.4 \%)$ in remission without medication ${ }^{21}$, while $29(10.9 \%)$ had inactive disease without fulfilling the remission criteria. Of the participants with inactive disease or in remission with/without medication, 38/162 (23.5\%) reported at least 1 
Table 1. Demographic and clinical characteristics by JIA categories in the TMJ study of the Nordic JIA cohort at the 17-year followup visit.

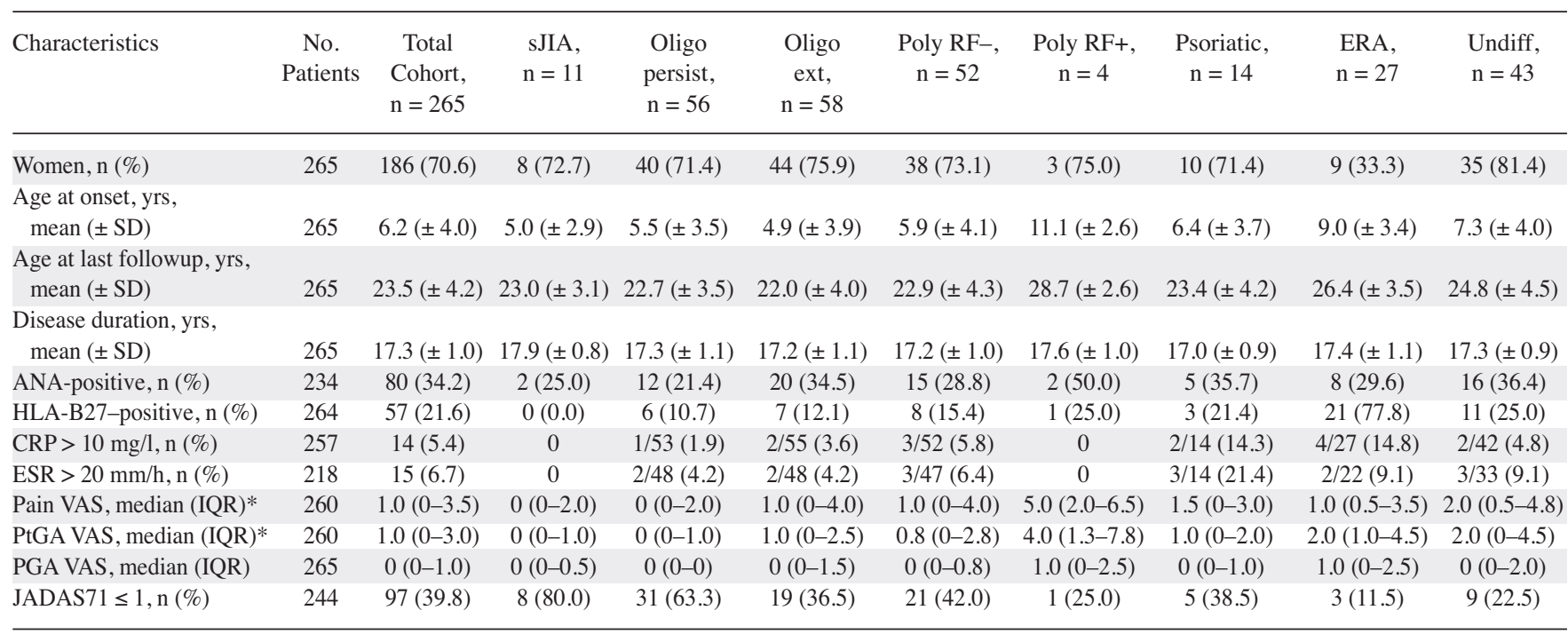

* Statistical significance $(\mathrm{p} \leq 0.05)$ among the JIA categories. PGA is reported if a followup visit was performed. JIA: juvenile idiopathic arthritis; TMJ: temporomandibular joint; sJIA: systemic JIA; oligo persist: persistent oligoarticular JIA; oligo ext: extended oligoarticular JIA; poly RF-: polyarticular rheumatoid factor-negative JIA; poly RF+: polyarticular RF-positive JIA; ERA: enthesitis-related arthritis; Undiff: undifferentiated JIA; ANA: antinuclear antibody; CRP: C-reactive protein; ESR: erythrocyte sedimentation rate; pain VAS: patient's intensity of pain on a 21-numbered circle visual analog scale (0-100); PtGA VAS: patient's global assessment of overall well-being on a 21-numbered circle VAS; PGA VAS: physician's global assessment of disease activity on a 21-numbered circle VAS; IQR: interquartile range; JADAS: Juvenile Arthritis Disease Activity Score.

Table 2. Prevalence of orofacial symptoms and dysfunction in the JIA group $(n=265)$ and the control group $(n=200)$.

\begin{tabular}{|c|c|c|c|c|}
\hline Variables & JIA Group & Control Group & Measures $(95 \% \mathrm{CI})$ & $\mathrm{p}$ \\
\hline \multicolumn{5}{|l|}{ Orofacial symptoms } \\
\hline No symptoms & $178(67.2)$ & $148(74.0)$ & & \\
\hline$\geq 1$ symptom & $87(32.8)$ & $52(26.0)$ & OR $1.4(0.91-2.14)$ & 0.11 \\
\hline TMJ pain & $65(24.5)$ & $14(7.0)$ & OR $4.32(2.30-8.60)$ & $<0.001^{*}$ \\
\hline TMJ morning stiffness & $42(15.8)$ & $9(4.5)$ & OR $4.02(1.86-9.60)$ & $<0.001^{*}$ \\
\hline Orofacial muscle pain & $74(27.9)$ & $44(22.0)$ & OR 1.07 (0.70-1.66) & 0.74 \\
\hline Limitation on chewing & $35(13.2)$ & $9(4.5)$ & OR $3.24(1.48-7.85)$ & $0.001^{*}$ \\
\hline Locking of the jaw & $20(7.5)$ & $17(8.5)$ & OR $0.88(0.42-1.84)$ & 0.71 \\
\hline \multicolumn{5}{|l|}{ Orofacial pain intensity } \\
\hline VAS pain > 0, median (IQR) & $25(5-85)$ & $19(6-60)$ & $Z=0.63$ & 0.53 \\
\hline \multicolumn{5}{|l|}{ Orofacial pain frequency } \\
\hline Pain frequency $>0$, median $(\mathrm{IQI}$ & R) $1(1-4)^{1}$ & $1(1-3)^{1}$ & $Z=2.61$ & $<0.01 *$ \\
\hline \multicolumn{5}{|c|}{ Orofacial pain profile } \\
\hline Pain index $>0^{2}$, median (IQR) & $30(5-260)$ & $20(6-120)$ & $\mathrm{Z}=1.53$ & 0.13 \\
\hline \multicolumn{5}{|l|}{ No. symptoms } \\
\hline Per subject, median (IQR) & $2(1-5)$ & $1(1-3)$ & $Z=2.26$ & $0.02 *$ \\
\hline \multicolumn{5}{|l|}{ Orofacial dysfunctions } \\
\hline No dysfunctions & $129(48.7)$ & $112(56.0)$ & & \\
\hline$\geq 1$ dysfunction & $136(51.3)$ & $88(44.0)$ & OR $1.34(0.91-1.97)$ & 0.12 \\
\hline Reduced $\mathrm{MIO}^{3}$ & $51(19.2)$ & $3(1.5)$ & OR $15.72(4.94-79.69)$ & $<0.001^{*}$ \\
\hline TMJ pain on palpation & $79(29.9)$ & $42(21.0)$ & OR $0.62(0.40-0.95)$ & $0.03 *$ \\
\hline Orofacial pain on palpation & $67(25.3)$ & $50(25.0)$ & OR $0.99(0.64-1.50)$ & 0.94 \\
\hline Asymmetric mouth opening & $29(10.9)$ & $32(16.0)$ & OR $0.65(0.36-1.15)$ & 0.11 \\
\hline \multicolumn{5}{|l|}{ No. dysfunctions } \\
\hline Per subject, median (IQR) & $1(0-2)$ & $0(0-2)$ & $\mathrm{Z}=2.34$ & $0.02 *$ \\
\hline
\end{tabular}


orofacial symptom, and 70 (43.2\%) had at least 1 clinical sign of dysfunction. In the same group, the frequencies of the reported symptoms were $38 / 162(23.5 \%)$ orofacial or jaw pain, $16(9.9 \%)$ morning stiffness in the jaws, $11(6.8 \%)$ limitation when chewing, and 7 (4.3\%) locking of the jaw. Further, a reduced MIO was found in 22/162 (13.6\%), TMJ pain on palpation in $37(22.8 \%)$, orofacial pain on palpation in $28(17.3 \%)$, and asymmetric mouth opening in $26(16.0 \%$; data not shown).

Radiologic findings. Normal CBCT of both TMJ was observed in 96/245 (39.2\%) of participants with JIA (Table $3)$. Thus, the prevalence of at least 1 abnormal radiological TMJ appearance was 149/245 (60.8\%) participants with JIA. Of these, 104/149 (69.8\%) had bilateral TMJ deformities. Of 253 joints with CBCT changes, $119(47.0 \%)$ had score 1 (deformity), 24 (9.5\%) score 2 (erosion), and 110 (43.5\%) score 3 (deformity and erosion). Abnormal condylar findings were most frequent in the rheumatoid factor-negative polyarticular group (76.5\%) and least frequent in the enthesitis-related arthritis (ERA) category (33.3\%), but TMJ deformities were present in all JIA categories (Table 4).
TMJ pain on palpation doubled the odds of having an abnormal TMJ on the CBCT scan (OR 2.1, 95\% CI 1.08-4.1), and there was a strong association with MIO $<-2$ SD (OR 7.5, 95\% CI 2.7-20.6) and abnormal TMJ on CBCT.

Of the $27(10.2 \%)$ participants having at least 1 intraarticular TMJ injection during the disease course, all had abnormal condylar findings on CBCT, and 17 (63.0\%) had erosions and deformities (data not shown).

Intraobserver reproducibility of the CBCT assessment. On 30 randomly selected CBCT examinations of $60 \mathrm{TMJ}$ assessed twice 6 months apart, intraobserver agreement was found in $53(88.3 \%)$ of the scans. Cohen's $\kappa=0.83$ indicated an almost perfect intraobserver agreement ${ }^{25}$.

Association between baseline predictors/clinical characteristics and abnormal radiologic condylar appearance at 17 years of followup. From the multivariate logistic regression analysis of the baseline predictors of condylar deformities/erosions, we found a positive association with an active joint count of $>4$ at baseline and a negative association with

Table 3. CBCT appearance of the condylar head of the left and right TMJ in the Nordic JIA cohort at the 17-year followup visit $(\mathrm{n}=245)$.

\begin{tabular}{lccccc}
\hline Right TMJ & Normal & Deformity & $\begin{array}{c}\text { Left TMJ } \\
\text { Erosion }\end{array}$ & Deformity and Erosion & Totaln \\
\hline Normal & $96(39.2)$ & $11(4.5)$ & $6(2.4)$ & $4(1.6)$ & $117(47.3)$ \\
Deformity & $12(4.9)$ & $29(11.8)$ & $2(0.8)$ & $17(6.9)$ & $60(24.5)$ \\
Erosion & $4(1.6)$ & $3(1.2)$ & $3(1.2)$ & $1(0.4)$ & $11(4.5)$ \\
Deformity/erosion & $8(3.3)$ & $16(6.5)$ & $2(0.8)$ & $31(12.7)$ & $58(23.7)$ \\
Total n & $120(49.0)$ & $59(24.1)$ & $13(5.3)$ & $53(21.6)$ & 245 \\
\hline
\end{tabular}

Values indicate $\mathrm{n}(\%)$ of joints. Each TMJ was scored as normal/abnormal and the abnormal joints were subsequently scored with deformity/erosion or with a combination of the 2 possibilities. The scoring system does not refer to a scale with gradual progression. Normal: normal condylar shape with smooth and intact outline/surface. Deformity: deformity of the condyle, with marked flattening or other changes in shape with smooth and intact outline/surface. Erosion: erosion of the condyle, with disruption of outline or uneven surface owing to cysts or erosion. Deformity and erosion: deformities plus erosive changes. CBCT: cone-beam computed tomography; TMJ: temporomandibular joints; JIA: juvenile idiopathic arthritis.

Table 4. Orofacial symptoms, dysfunctions, and temporomandibular radiological appearance according to JIA category $(\mathrm{n}=245)$.

\begin{tabular}{|c|c|c|c|c|c|c|c|c|c|}
\hline Variables & $\begin{array}{l}\text { Total Cohort, } \\
\mathrm{n}=245\end{array}$ & $\begin{array}{l}\text { sJIA, } \\
\mathrm{n}=10\end{array}$ & $\begin{array}{l}\text { Oligo persist, } \\
\mathrm{n}=52\end{array}$ & $\begin{array}{l}\text { Oligo ext, } \\
\mathrm{n}=53\end{array}$ & $\begin{array}{l}\text { Poly RF- } \\
\mathrm{n}=51\end{array}$ & $\begin{array}{c}\text { Poly RF+, } \\
n=4\end{array}$ & $\begin{array}{l}\text { Psoriatic, } \\
\mathrm{n}=13\end{array}$ & $\begin{array}{l}\text { ERA, } \\
\mathrm{n}=24\end{array}$ & $\begin{array}{l}\text { Undiff, } \\
\mathrm{n}=38\end{array}$ \\
\hline TMJ-reported pain & $65(26.5)$ & $2(20.0)$ & $8(15.4)$ & $14(26.4)$ & $12(23.5)$ & $1(25.0)$ & $4(30.8)$ & $10(41.7)$ & $14(36.8)$ \\
\hline Chewing limitations & $35(14.3)$ & $0(0.0)$ & $5(9.6)$ & $6(11.3)$ & $8(15.7)$ & $1(25.0)$ & $3(23.1)$ & $4(16.7)$ & $8(21.1)$ \\
\hline $\mathrm{MIO}<2 \mathrm{SD}$ & $51(20.8)$ & $0(0.0)$ & $5(9.6)$ & $14(26.4)$ & $13(25.5)$ & $1(25.0)$ & $2(15.4)$ & $5(20.8)$ & $11(28.9)$ \\
\hline TMJ pain on palpation & $59(24.1)$ & $1(10.0)$ & $8(15.4)$ & $13(24.5)$ & $12(23.5)$ & $1(25.0)$ & $4(30.8)$ & $4(16.7)$ & $16(42.1)$ \\
\hline $\begin{array}{l}\text { Radiological deformities } \\
\text { and/or erosions }\end{array}$ & $149(60.8)$ & $5(50.0)$ & $26(50.0)$ & $36(67.9)$ & $39(76.5)$ & $3(75.0)$ & $8(61.5)$ & $8(33.3)$ & $24(63.2)$ \\
\hline
\end{tabular}

Values indicate $\mathrm{n}(\%)$ of joints. JIA: juvenile idiopathic arthritis; sJIA: systemic JIA; oligo persist: persistent oligoarticular JIA; oligo ext: extended oligoarticular JIA; poly RF-: polyarticular rheumatoid factor-negative JIA; poly RF+: polyarticular RF-positive JIA; ERA: enthesitis-related arthritis; Undiff: undifferentiated JIA; TMJ: temporomandibular joint; MIO: maximal incisal opening. 
Table 5. Associations between clinical characteristics at baseline, 8-year followup, or at any time during the disease course, and condylar deformities/erosions at the 17-year followup.

\begin{tabular}{|c|c|c|}
\hline Variables & Multivariate OR $(95 \%$ CI) & $\mathrm{p}$ \\
\hline \multicolumn{3}{|l|}{ Predictors early in disease course, $n=211$} \\
\hline Age at onset $<6$ yrs & $1.01(0.52-1.97)$ & 0.97 \\
\hline Female sex & $1.08(0.54-2.17)$ & 0.84 \\
\hline Active joint count at baseline visit $>4$ joints & $2.14(1.11-4.18)$ & $0.02 *$ \\
\hline ESR $>20$ at baseline & $1.88(0.95-3.70)$ & 0.07 \\
\hline HLA-B27-positive & $0.44(0.20-0.98)$ & $0.04^{*}$ \\
\hline Uveitis at baseline & $1.54(0.48-4.99)$ & 0.47 \\
\hline \multicolumn{3}{|l|}{ Predictors at 8 years of followup, $n=234$} \\
\hline Age at onset $<6$ yrs & $1.31(0.73-2.36)$ & 0.11 \\
\hline Female sex & $1.31(0.60-2.13)$ & 0.36 \\
\hline Cumulative active joint count $>4$ & $2.07(1.05-4.09)$ & $0.03^{*}$ \\
\hline $\mathrm{JADI}-\mathrm{A}>0$ & $1.66(0.94-2.93)$ & 0.08 \\
\hline MTX & $1.22(0.62-2.41)$ & 0.62 \\
\hline Biologics & $2.10(0.75-5.86)$ & 0.16 \\
\hline ERA & $0.35(0.12-0.97)$ & $0.04^{*}$ \\
\hline \multicolumn{3}{|l|}{ Associations during the disease course $* *, n=244$} \\
\hline Age at onset $<6$ yrs & $1.18(0.65-2.14)$ & 0.47 \\
\hline Female sex & $0.78(0.40-1.53)$ & 0.47 \\
\hline DMARD during disease course & $1.18(0.59-2.36)$ & 0.60 \\
\hline Biologics during disease course & $2.37(1.18-4.74)$ & $0.02 *$ \\
\hline Cumulative active joints $>4$ at $17-y r$ followup & $1.94(0.99-3.80)$ & 0.06 \\
\hline Orofacial dysfunction at 17-yr followup & $3.13(1.74-5.62)$ & $<0.01 *$ \\
\hline ERA & $0.17(0.06-0.50)$ & $0.01 *$ \\
\hline
\end{tabular}

\footnotetext{
* Statistical significance $(\mathrm{p} \leq 0.05)$. ** Associations between clinical characteristics at any time of the disease course and condylar deformities/erosions. ESR: erythrocyte sedimentation rate; JADI-A: Juvenile Arthritis Damage Index-Articular; MTX: methotrexate; ERA: enthesitis-related arthritis; DMARD: disease-modifying antirheumatic drugs.
}

the presence of HLA-B27 (Table 5). At the 8-year followup, a cumulative joint count of $>4$ and the ERA category was associated with condylar changes. Logistic regression analysis on age at onset and sex did not show any association with condylar deformity.

Treatment with biologics during the disease course and orofacial dysfunctions 17 years after onset were associated with a significantly higher risk of TMJ deformity $(\mathrm{p}=0.02$ and $\mathrm{p}<0.01$, respectively). The ERA category had a significant lower risk of developing TMJ deformities (Table 5).

\section{DISCUSSION}

To our knowledge, this is the first study to report longterm data on temporomandibular symptoms, dysfunctions, and radiological findings in a population-based JIA cohort from the early biologic era compared to controls. The results showed higher prevalence of TMJ pain, TMJ morning stiffness, and chewing limitations in the JIA group. We found a higher prevalence of TMJ pain on palpation and a reduced MIO among the JIA participants; however, the prevalence of orofacial pain on palpation and asymmetric mouth opening was not different from that seen in the control group. The control group's complaints were mild and comparable to what has been reported in TMD unrelated to arthritis ${ }^{26}$.

It is difficult to compare our results with previous longterm TMJ-related outcome studies from the prebiologic era because of differences in study designs, terminologies, and definitions. In a questionnaire-based cohort study of 28 patients with JIA, Engstrøm, et al ${ }^{13}$ documented a higher risk of TMJ pain and dysfunction in patients with JIA than in healthy controls 15 years after diagnosis, with $7 \%$ reporting limitation on chewing and $39 \%$ facial or TMJ pain, which is close to our findings. In 2001, Bakke, et al ${ }^{12}$ conducted a 26-year followup study on 42 women with JIA, of whom $17 \%$ experienced orofacial pain. None of the 21 healthy controls reported orofacial pain. In a more recent crosssectional 17-year followup study by Resnick and colleagues ${ }^{4}$, $24 \%$ of the 21 patients with JIA reported TMJ pain, which is consistent with our findings.

Our study showed no difference in self-reported orofacial muscle pain. Previous studies on TMD have consistently documented that orofacial pain is a common finding in healthy adults. The American OPPERA study ${ }^{27}$ enrolled 4346 adults and demonstrated a $4 \%$ annual incidence of painful TMD, and the prevalence of TMJ and/or facial pain was 19\%. In comparison, the frequency of TMJ and/or facial pain in our control group was $33 \%$. This difference may be explained by an older mean age in the OPPERA study, considering a peak incidence of TMJ/facial pain in the age group 25-34 years ${ }^{28}$.

It is not possible to differentiate JIA-associated TMD from

Personal non-commercial use only. The Journal of Rheumatology Copyright @ 2020 . All rights reserved. 
other TMD etiologies based on clinical examination. We can only hypothesize that TMD symptoms and dysfunctions reported in the JIA group originate from TMJ involvement.

Few studies on longterm outcome of TMJ dysfunctions have been published, and dissimilarities in the terminologies used hamper comparison. Prevalence of reduced MIO was $19-29 \%$ in 2 previous longterm studies ${ }^{4,12}$, which matches our findings. TMJ pain on palpation was demonstrated in $17 \%^{12}$, which is lower than our prevalence (30\%); however, that study was small and the methods used were not the same as ours.

Unexpectedly, we found the same rate of asymmetric mouth opening in the JIA and the control group. No previous longterm studies have reported an asymmetric mouth opening for comparison. Our findings can partly be explained by the high prevalence of bilateral CBCT-verified deformities in the JIA group, albeit such deformities do not necessarily lead to asymmetric mouth opening. Our findings could also suggest that asymmetric mouth opening can normalize over time. It is also well known that in clinical examinations, deflection can be misinterpreted as asymmetric mouth opening, leading to an uncertain measurement of the latter. The JIA participants and the control group were not evaluated by the same orthodontists, which can also influence the results.

A large number of patients experienced continued TMJ symptoms/dysfunctions despite inactive disease/remission, indicating a need for continued, standardized orofacial monitoring 17 .

CBCT is a well-established method to detect TMJ deformity and hard tissue changes. Recently, Kellenberger, et $a^{29}$ suggested magnetic resonance imaging (MRI) for monitoring TMJ arthritis and published an additive scoring system to assess the osseous deformities. However, it was not published at the beginning of our study, and CBCT has previously been used for assessment of hard tissue changes ${ }^{4,30}$. In total, about $61 \%$ of the JIA participants had at least 1 condyle with abnormal CBCT findings, and about $70 \%$ of these subjects had bilateral changes. Importantly, the presence of radiographic condylar changes does not necessarily imply the presence of dentofacial deformity. The association between radiographic TMJ changes and dentofacial deformity will be established in a future study.

Using a different CBCT scoring system, Resnick, et al found TMJ abnormalities in 55\% of their patients with JIA at the age of 26 years, of whom $79 \%$ had bilateral changes ${ }^{4}$. We found a slightly higher prevalence of radiographic TMJ changes. However, we have to acknowledge that some of the abnormal findings may not be directly attributable to the JIA disease because abnormal CBCT findings of the TMJ have been described in healthy individuals. Using a CBCT scoring system similar to the one used here, Stoustrup, et al reported that 5/19 (26\%) healthy controls had abnormal TMJ appearance ${ }^{30}$; and Kellenberger, et $a l^{31}$ showed that anterior disk displacement in non-JIA adolescents most often is associated with osseous TMJ changes, mainly erosions, in
non-JIA individuals. For ethical reasons, CBCT examinations of the TMJ were not performed in the healthy control group.

Study heterogeneity and small sample size have compromised previous studies of predictors of TMJ involvement ${ }^{32}$. However, our finding of a lower risk of condylar changes with the presence of HLA-B27 has also been observed in previous studies ${ }^{2,33}$. Likewise, an active joint count of more than 4 joints as a predictor of TMJ involvement is consistent with the findings in previous, smaller studies ${ }^{33,34}$. The protective effect of the ERA category for developing condylar deformities and/or erosions is a new finding. A high risk of developing condylar changes was associated with a severe disease course, indicated by the use of biologic treatment and a cumulative joint count of more than 4. It is highly unlikely that there is a causal relationship between biologic treatment and development of condylar changes. Instead, the association observed likely reflects the use of biologics in more severe disease.

The strength of our study is the population-based setting, which qualifies it as a nonselected JIA cohort study. Further, the generalizability of the study gains from the use of the consensus-based recommendations for clinical orofacial examination and the use of contemporary terminology regarding orofacial findings in JIA, which promotes comparison of our findings with those of future studies. Our study is based on an ethnically homogeneous Nordic cohort; still, the distribution of the JIA categories is consistent with other European and North American cohorts ${ }^{35}$.

However, several limitations must be acknowledged. Of the total cohort, $37 \%$ was lost to followup. Age at onset was lower in the included group, but we found no differences in sex, disease activity at baseline, and JIA category. Considering the fact that most of the participants are not attending pediatric clinics anymore, the response rate after 17 years was considered acceptable.

The CBCT scans applied to evaluate condylar changes have also been used previously ${ }^{30,36}$ but do not discriminate graduation of severity. In addition, no knowledge about the time-related association between the development of the erosions and deformities can be provided. Because MRI was not performed, we cannot draw conclusions on TMJ arthritis activity at the last visit. Further, repeated MRI were not done routinely, but might have influenced the choice of treatment for the participants on whom it was performed, and accordingly have affected the outcome.

Our findings indicate that extensive symptoms and dysfunctions are seen in JIA even 17 years after disease onset, even in patients registered with inactive disease/remission. Individuals with substantial condylar damage were found in all JIA categories. We suggest including aspects of TMJ involvement in the general clinical decision making by including orofacial symptom and dysfunction assessment as an integrated part of general health assessment in JIA, guided by recent consensus-based recommendations ${ }^{17}$. 


\section{ACKNOWLEDGMENT}

We thank the patients, coordinators, and investigators of the Nordic Study Group of Pediatric Rheumatology.

\section{REFERENCES}

1. Weiss PF, Arabshahi B, Johnson A, Bilaniuk LT, Zarnow D, Cahill $\mathrm{AM}$, et al. High prevalence of temporomandibular joint arthritis at disease onset in children with juvenile idiopathic arthritis, as detected by magnetic resonance imaging but not by ultrasound. Arthritis Rheum 2008;58:1189-96.

2. Cannizzaro E, Schroeder S, Muller LM, Kellenberger CJ, Saurenmann RK. Temporomandibular joint involvement in children with juvenile idiopathic arthritis. J Rheumatol 2011;38:510-5.

3. Stoll ML, Sharpe T, Beukelman T, Good J, Young D, Cron RQ. Risk factors for temporomandibular joint arthritis in children with juvenile idiopathic arthritis. J Rheumatol 2012;39:1880-7.

4. Resnick CM, Dang R, Henderson LA, Zander DA, Daniels KM, Nigrovic PA, et al. Frequency and morbidity of temporomandibular joint involvement in adult patients with a history of juvenile idiopathic arthritis. J Oral Maxillofac Surg 2017;75:1191-200.

5. Kuseler A, Pedersen TK, Gelineck J, Herlin T. A 2 year followup study of enhanced magnetic resonance imaging and clinical examination of the temporomandibular joint in children with juvenile idiopathic arthritis. J Rheumatol 2005;32:162-9.

6. Pedersen TK, Kuseler A, Gelineck J, Herlin T. A prospective study of magnetic resonance and radiographic imaging in relation to symptoms and clinical findings of the temporomandibular joint in children with juvenile idiopathic arthritis. J Rheumatol 2008;35:1668-75.

7. Enlow DH, Hans MG. Growth of the mandible. In: Essentials of facial growth. Philadelphia: Saunders; 1996:57-78.

8. Larheim TA, Haanaes HR, Dale K. Radiographic temporomandibular joint abnormality in adults with micrognathia and juvenile rheumatoid arthritis. Acta Radiol Diagn 1981;22:495-504.

9. Fjeld M, Arvidsson L, Smith HJ, Flato B, Ogaard B, Larheim T. Relationship between disease course in the temporomandibular joints and mandibular growth rotation in patients with juvenile idiopathic arthritis followed from childhood to adulthood. Pediatr Rheumatol Online J 2010;8:13.

10. Koos B, Gassling V, Bott S, Tzaribachev N, Godt A. Pathological changes in the TMJ and the length of the ramus in patients with confirmed juvenile idiopathic arthritis. J Craniomaxillofac Surg 2014;42:1802-7.

11. Frid P, Nordal E, Bovis F, Giancane G, Larheim TA, Rygg M, et al; Paediatric Rheumatology International Trials Organisation. Temporomandibular joint involvement in association with quality of life, disability, and high disease activity in juvenile idiopathic arthritis. Arthritis Care Res 2017;69:677-86.

12. Bakke M, Zak M, Jensen BL, Pedersen FK, Kreiborg S. Orofacial pain, jaw function, and temporomandibular disorders in women with a history of juvenile chronic arthritis or persistent juvenile chronic arthritis. Oral Surg Oral Med Oral Pathol Oral Radiol Endod 2001;92:406-14.

13. Engstrøm AL, Wanman A, Johansson A, Keshishian P, Forsberg M. Juvenile arthritis and development of symptoms of temporomandibular disorders: a 15-year prospective cohort study. J Orofac Pain 2007;21:120-6.

14. Arvidsson LZ, Fjeld MG, Smith HJ, Flato B, Ogaard B, Larheim TA. Craniofacial growth disturbance is related to temporomandibular joint abnormality in patients with juvenile idiopathic arthritis, but normal facial profile was also found at the 27-year follow-up. Scand J Rheumatol 2010;39:373-9.

15. Berntson L, Andersson Gare B, Fasth A, Herlin T, Kristinsson J, Lahdenne P, et al; Nordic Study Group. Incidence of juvenile idiopathic arthritis in the Nordic countries. A population based study with special reference to the validity of the ILAR and EULAR criteria. J Rheumatol 2003;30:2275-82.

16. Nordal E, Zak M, Aalto K, Berntson L, Fasth A, Herlin T, et al; Nordic Study Group of Pediatric Rheumatology. Ongoing disease activity and changing categories in a long-term Nordic cohort study of juvenile idiopathic arthritis. Arthritis Rheum 2011;63:2809-18.

17. Stoustrup P, Twilt M, Spiegel L, Kristensen KD, Koos B, Pedersen TK, et al; euroTMjoint Research Network. Clinical orofacial examination in juvenile idiopathic arthritis: international consensus-based recommendations for monitoring patients in clinical practice and research studies. J Rheumatol 2017;44:326-33.

18. Petty RE, Southwood TR, Manners P, Baum J, Glass DN, Goldenberg J, et al; International League of Associations for Rheumatology. International League of Associations for Rheumatology classification of juvenile idiopathic arthritis: second revision, Edmonton, 2001. J Rheumatol 2004;31:390-2.

19. Consolaro A, Ruperto N, Bazso A, Pistorio A, Magni-Manzoni S, Filocamo G, et al; Paediatric Rheumatology International Trials Organisation. Development and validation of a composite disease activity score for juvenile idiopathic arthritis. Arthritis Rheum 2009;61:658-66.

20. Consolaro A, Bracciolini G, Ruperto N, Pistorio A, Magni-Manzoni S, Malattia C, et al; Paediatric Rheumatology International Trials Organization. Remission, minimal disease activity, and acceptable symptom state in juvenile idiopathic arthritis: defining criteria based on the juvenile arthritis disease activity score. Arthritis Rheum 2012;64:2366-74.

21. Wallace CA, Ruperto N, Giannini E; Childhood Arthritis and Rheumatology Research Alliance; Pediatric Rheumatology International Trials Organization; Pediatric Rheumatology Collaborative Study Group. Preliminary criteria for clinical remission for select categories of juvenile idiopathic arthritis. J Rheumatol 2004;31:2290-4.

22. Stoustrup P, Resnick CM, Pedersen TK, Abramowicz S, Michelotti A, Küseler A; TMJ Juvenile Arthritis Working Group. Standardizing terminology and assessment for orofacial conditions in juvenile idiopathic arthritis: international, multidisciplinary consensus-based recommendations. J Rheumatol 2019;46:518-22.

23. Beaton DE, Bombardier C, Guillemin F, Ferraz MB. Guidelines for the process of cross-cultural adaptation of self-report measures. Spine 2000;25:3186-91.

24. Stoustrup P, Kristensen KD, Kuseler A, Herlin T, Pedersen TK. Normative values for mandibular mobility in Scandinavian individuals 4-17 years of age. J Oral Rehabil 2016;43:591-7.

25. McHugh ML. Interrater reliability: the kappa statistic. Biochem Med 2012;22:276-82.

26. Slade GD, Bair E, Greenspan JD, Dubner R, Fillingim RB, Diatchenko L, et al. Signs and symptoms of first-onset TMD and sociodemographic predictors of its development: the OPPERA prospective cohort study. J Pain 2013;12 Suppl:T20-32.

27. Slade GD, Ohrbach R, Greenspan JD, Fillingim RB, Bair E, Sanders $\mathrm{AE}$, et al. Painful temporomandibular disorder: decade of discovery from OPPERA studies. J Dent Res 2016;95:1084-92.

28. Isong U, Gansky SA, Plesh O. Temporomandibular joint and muscle disorder-type pain in U.S. adults: the National Health Interview Survey. J Orofac Pain 2008;22:317-22.

29. Kellenberger CJ, Junhasavasdikul T, Tolend M, Doria AS. Temporomandibular joint atlas for detection and grading of juvenile idiopathic arthritis involvement by magnetic resonance imaging. Pediatr Radiol 2018;48:411-26.

30. Stoustrup PB, Ahlefeldt-Laurvig-Lehn N, Kristensen KD, Arvidsson LZ, Twilt M, Cattaneo PM, et al. No association between types of unilateral mandibular condylar abnormalities and facial asymmetry in orthopedic-treated patients with juvenile idiopathic arthritis. Am J Orthod Dentofacial Orthop 2018;153:214-23. 
31. Kellenberger CJ, Bucheli J, Schroeder-Kohler S, Saurenmann RK, Colombo V, Ettlin DA. Temporomandibular joint magnetic resonance imaging findings in adolescents with anterior disk displacement compared to those with juvenile idiopathic arthritis. J Oral Rehabil 2019;46:14-22.

32. Kristensen KD, Stoustrup P, Kuseler A, Pedersen TK, Twilt M, Herlin T. Clinical predictors of temporomandibular joint arthritis in juvenile idiopathic arthritis: a systematic literature review. Semin Arthritis Rheum 2016;45:717-32.

33. Pedersen TK, Jensen JJ, Melsen B, Herlin T. Resorption of the temporomandibular condylar bone according to subtypes of juvenile chronic arthritis. J Rheumatol 2001;28:2109-15.
34. Twilt M, Mobers SM, Arends LR, ten Cate R, van Suijlekom-Smit L. Temporomandibular involvement in juvenile idiopathic arthritis. J Rheumatol 2004;31:1418-22.

35. Consolaro A, Ravelli A. Unraveling the phenotypic variability of juvenile idiopathic arthritis across races or geographic areas - key to understanding etiology and genetic factors? J Rheumatol 2016;43:683-5.

36. Hatcher D. Progressive condylar resorption: pathologic processes and imaging considerations. Semin Orthod 2013;19:97-105. 